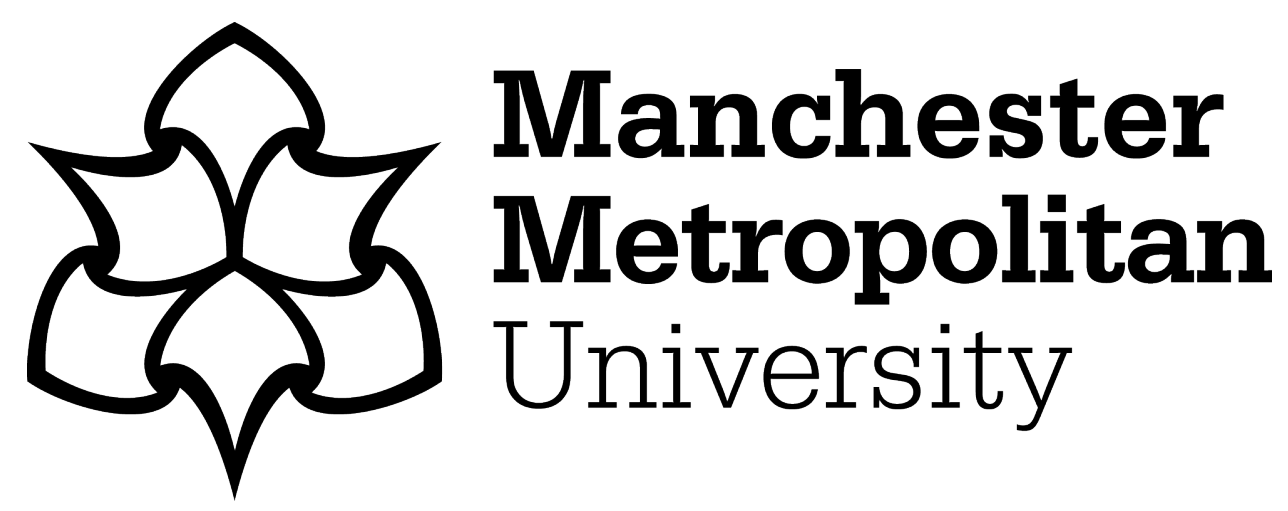

Mazidi, N, Roobottom, C and Masala, G ORCID logoORCID: https://orcid.org/0000-0001-6734-9424 (2019) Automatic Quantification of Breast Arterial Calcification on Mammographic Images. In: Innovation in Medincine \& Heathcare, 17 June 2019 - 19 June 2019, St Julians, Malta.

Downloaded from: https://e-space.mmu.ac.uk/623234/

Version: Accepted Version

Publisher: Springer Nature

DOI: https://doi.org/10.1007/978-981-13-8566-7_28

Please cite the published version 


\title{
Automatic Quantification of Breast Arterial Calcification on Mammographic Images
}

\author{
Najim Mazidi ${ }^{1}$, Carl Roobottom ${ }^{2}$ and Giovanni Masala ${ }^{3}$ \\ ${ }^{1}$ University of Plymouth, Plymouth UK \\ ${ }^{2}$ Peninsula Schools of Medicine and Dentistry, University of Plymouth, Plymouth UK \\ ${ }^{3}$ Manchester Metropolitan University, Manchester, UK
}

\begin{abstract}
This paper describes the research and development of an automatic computer system that is used to quantify breast arterial calcifications in mammography scans. A few prior studies have attempted to establish a relationship between breast arterial calcification (BAC) and the rate of coronary artery disease $(\mathrm{CAD})$ risk factors. The majority of these studies demonstrated a positive association between BAC and increasing age. Large scale cohort studies and retrospective studies have almost uniformly suggested a strong association between BAC and cardiovascular disease related morbidity and mortality. This strong association of BAC with cardiovascular pathology suggests that BAC should also be persistently associated with radiographically determined CAD. A method of image processing, segmentation and quantification used to highlight and recognise calcified blood vessels in the breast is proposed and described in detail. This project aims to introduce a new use for digital Mammography, which is currently solely used for diagnosing breast cancer in female patients.

A method of detecting BAC is introduced at no additional cost, having an adequate degree of accuracy, around $82 \%$, which means that this type of system could be used to assist a radiographer in diagnosing BAC by indicating whether the patient has a high or low severity of calcification.
\end{abstract}

Keywords: Breast Arterial Calcification, CAD, medical imaging.

\section{Introduction}

Coronary artery disease (CAD) is a major cause of mortality and morbidity in women, accounting for over a third of female deaths around the world [1], and it is often not detected until it is too late. Overall, more American women than men die of CAD annually, and until the age of 80 years, women who suffer from acute coronary syndrome suffer higher mortality rates. Various causes postulated to account for the difference in CAD related mortality include decreased disease detection in women and gender differences in symptomatology and pathophysiology. Early detection and treatment of coronary artery disease remain underutilized among women [1-3].

Breast arterial calcification (BAC) is a calcium deposition in the media of the peripheral arterioles, which is known as Monckeberg medial calcific sclerosis [2], and it 
is regularly found in mammography. Mammography is a process of using low-energy $\mathrm{X}$-rays to examine the human breast and it is a common, and widely accepted, method of screening for breast cancer. BAC can also be detected in mammograms, but it is rarely included in final reports because it is considered a benign finding that is not relevant to the diagnosis of breast cancer.

There is increasing evidence that breast arterial calcification (BAC) is a good indicator of a person being in risk of cardiovascular disease (CVD) [2,4]. However, as BACs visible in mammograms are usually ignored, these findings are wasted. Mammography equipment and expertise costs a substantial amount of money and by only having one use for it, it becomes difficult for hospitals and patients to afford this.

A few prior studies have attempted to establish a relationship between BAC and $\mathrm{CAD}$ risk factors. The majority of these studies demonstrated a positive association between BAC and increasing age [1-3]. Large scale cohort studies, and retrospective studies, have almost uniformly suggested a strong association between BAC and cardiovascular disease related morbidity and mortality. This strong association of BAC with cardiovascular pathology suggests that BAC should also be persistently associated with radiographically determined CAD. The gold standard of CAD determination has historically been catheter coronary angiography. Screening mammography is a widely used diagnostic test to detect breast cancer. If BAC could serve as a marker for $\mathrm{CAD}$, then screening mammography could yield added value as a single test already in wide use for the additional detection of $\mathrm{CAD}$, an additional highly prevalent disease with high morbidity and mortality.

Dual source coronary computed tomography angiography (CTA) has been shown to be effective for non-invasive diagnosis of CAD based on the demonstration of calcified, and noncalcified, coronary arterial plaque and grading of stenotic disease.

A study on PLoS One [4] was conducted to assess the relationship between mammographic BAC and CAD based on coronary CTA findings. Medical records for 100 patients who received both a mammogram and a coronary CTA were reviewed for imaging findings and CAD risk factors. Correlation was measured between BAC and $\mathrm{CAD}$ and logistic regression was used to create a classifier for predicting CAD based on BAC and common risk factors. However, nobody shows results on big dataset and there are some criticisms on this [5]. There are few works that try to solve the problem of the calcifications measure in the breast vessel using automatic systems. Searching in the literature, it is possible to find interest on developing a specific automatic BAC to measure the vessels calcification, but this is an open issue and few papers present preliminary results on it $[6,7]$.

The next sections are articulated as follow; in section 2 the related works are presented, in section 3 the method is explained, in section 4 the dataset is illustrated, in section 5 the results are discussed and finally the conclusions are made in the last section. 


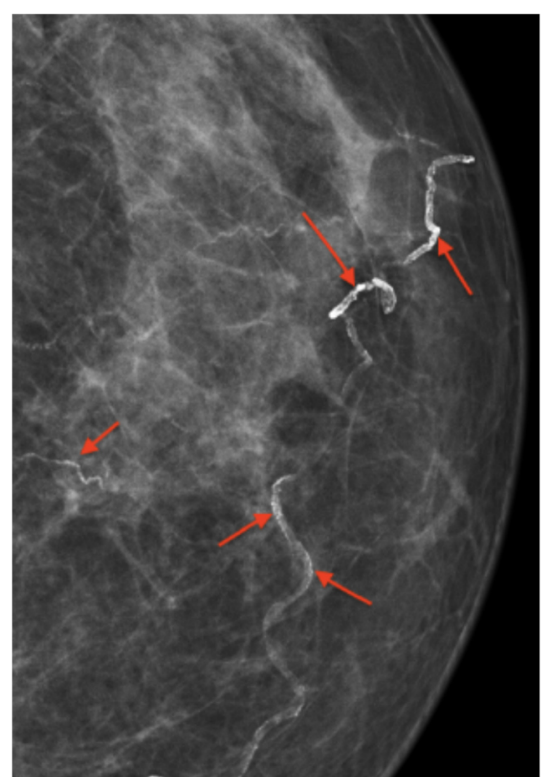

Fig. 1. Mammogram image showing a high grade of calcification. Red arrows indicate the vessels that are calcified and affected by BAC.

\section{Previous Research}

Some previous research has been done into finding a way to classify and quantify arterial calcification on digital mammograms and some partial solutions have been suggested. Although solutions have been put forward for classifying BACs, no solution has been found to fully quantify them automatically or suggest a correlation between BAC and CAD.

An important study into this subject was made by Nava et al. [6], which we used as an initial guidance for this paper. The authors in [6] proposed two steps; edge detection and image segmentation. A line-strength algorithm is used to process the image in order to make the vessels more visible and highlighted, removing unnecessary pixels. A region growing algorithm is used to highlight pixels that may have been ignored by using line strength; these pixels are usually located inside the walls of the vessels. The findings show that the methods proposed by Nava et al. can detect and segment BAC, with enough precision, in order to be able to accurately quantify breast arterial calcifications in the breast region.

Another study was carried out by Cheng et al.[7], which proposes a random walks algorithm that could be used to extract calcified vessels from mammograms. The solution proposed in [7] consists of two steps; a random 'walk' pixel tracking and a compiling and linking step. The random-walk step involves tracking of the traversing direction and jumping distance, and 'vesselness' value of each pixel when traversing the image, in order to generate possible paths. The second step involves organising and grouping all paths into 'calcified vessel tracts'. The result shown in [7] demon- 
strated that the performance of the algorithm is close to that of manual human diagnosis. Ge et al.[8] have proposed a way of detecting calcified vessels using clues that are given by calcification. They applied a principle curve clustering method on the mammogram in order detect calcified 'segments'. The main problem with this method is that if a mammogram has multiple segments, the segments will not be grouped together. This means that we are not able to see the length of a vessel, which creates a problem when trying to measure and evaluate severity of calcifications.

During their study on BACs, as possible marker for cardiovascular disease, Iribarrenand and Molloi have identified 17 studies that have been published that show a link between breast arterial calcification and clinical or sub-clinical cardio-vascular disease [9]. In total there are 4 prospective and 13 cross-sectional studies. These studies are shown on table 1. All the studies show a link between the presence of BAC and cardiovascular disease (CVD), be it fatal or non-fatal.

Table 1. Published studies on the association between BAC and CVD, and their outcomes [9].

\begin{tabular}{lll}
\hline Author & Sample Size & Outcome(s) \\
\hline Van Noord et al [10] & 12,239 & TIA/Stroke, thrombosis, myocardial infarction \\
Kemmeren et al. & 12,239 & CVD death, total mortality \\
Iribarren et al. & 12,761 & $\begin{array}{l}\text { Incident fatal or non-fatal CHD, ischemic } \\
\text { stroke, heart failure }\end{array}$ \\
Schnatz et al. & 1,454 & Incident CHD in 5 years \\
Doerger et al. & 1,803 & Angiographic CAD \\
Fiuza Ferreira et al. & 131 & Angiographic CAD \\
Henkin et al. & 319 & Angiographic CAD \\
Topal et al. & 123 & Angiographic CAD \\
Kataoka et al. & 1,590 & Prevalent CHD \\
Maas et al. & 499 & Coronary Artery calcification \\
Dale et al. & 645 & Peripheral vascular disease \\
Dale et al. & 1,000 & Self-reported history of CAD \\
Yildiz et al. & 54 & Carotid IMT \\
Ferreira et al. & 307 & Global CVD \\
Oliveira et al. & 80 & Clinical CAD \\
Sedighi et al. & 537 & IMT and carotid plaque by ultrasound \\
Ahn et al. & 168 & WMH and PVH on Brain MRI \\
& & \\
\hline
\end{tabular}




\section{Method}

The presented approach starts with the image preparation, removing black space surrounding breast region from image. The resolution of the image is also lowered, because the original images are very large. A further Gaussian filter is used to blur and smooth the image before processing.

The Line Strength algorithm is used to highlight and extract linear features in a mammogram, in a similar way to the methods proposed by Zwiggelaar et al. [11] and Nava et al. [6]. A value that reflect the strength of a line $S$ is calculated for each pixel:

$$
\mathrm{S}=\alpha(\mathrm{L}-\mathrm{N})+(1-\alpha)(\mathrm{I}-\mathrm{G})
$$

where $\alpha$ is a constant between 0 and $1, \mathrm{~L}$ is the average pixel grey value for the line passing through the pixel (in an orientation that produces the highest value), $\mathrm{N}$ is the average pixel grey value inside the specified square neighbourhood around the selected pixel, I is the original pixel grey value of the selected pixel, and $G$ is the resulting pixel grey value from passing the image through a Gaussian filter. The purpose of this algorithm is to extract a skeletonized structure of the linear parts of the mammographic image. The result $\mathrm{S}$ of the Line Strength algorithm is then fed into a thresholding function, which compares each pixel with a given threshold value, in order to extract the linear structures with the highest intensity. Pixels with high intensity have a higher probability to be a calcification.

Afterward a region growing algorithm [12] examines a given pixel, and the neighbourhood around it, and determines whether they are similar enough to the seed to be added to the same region. Region growing is a region-based and pixel-based method for image segmentation. This approach starts with a seed pixel, examining the neighbourhood around it, and determined whether they are similar enough to the seed to be added to the same region. It is then iterated until the threshold is met for the grown region or no more pixels meet the criteria to be added to the region. The pixels, which are added to the region, are usually located inside the calcified vessels.

Once the image has analysed by the region growing algorithm, an average white level for the whole image is calculated. In order to obtain this value, a mean of all pixel grey values of the image is calculated. In addition, a white intensity is calculated for the whole image by only obtaining the mean grey level of the activated pixels (non black). The average white level and average white intensity are then multiplied together. Such white level is then used to estimate the presence and grade of calcification. In figure 2 an overview of the steps illustrated is made. 


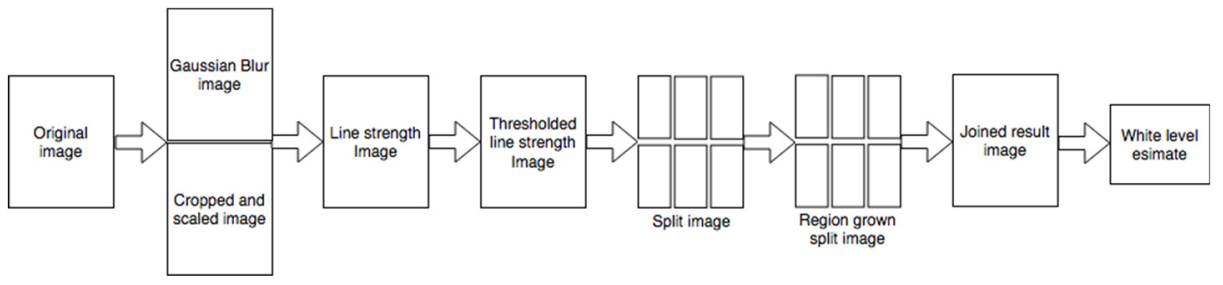

Fig. 2. Steps of processing, segmenting and classifying a radiological image of the breast.

\section{Dataset}

The dataset used in this project consists of four mammograms for each of the 26 patients, having a total of 104 radiological images, and it is provided by the Peninsula Radiology Academy in Plymouth, UK. The patients are classified based on the grade of calcification between 1 and 4 . Grade 4 is a group of five patients while the other groups are composed by seven patients. The data does not contain anything that could be used to identify the patients, therefore it is completely anonymous.

\section{Results}

The figure 3 shows a comparison of mammographic image in all stages, from original to region grown. This shows the progress that the image goes through in order to be able to identify calcifications.

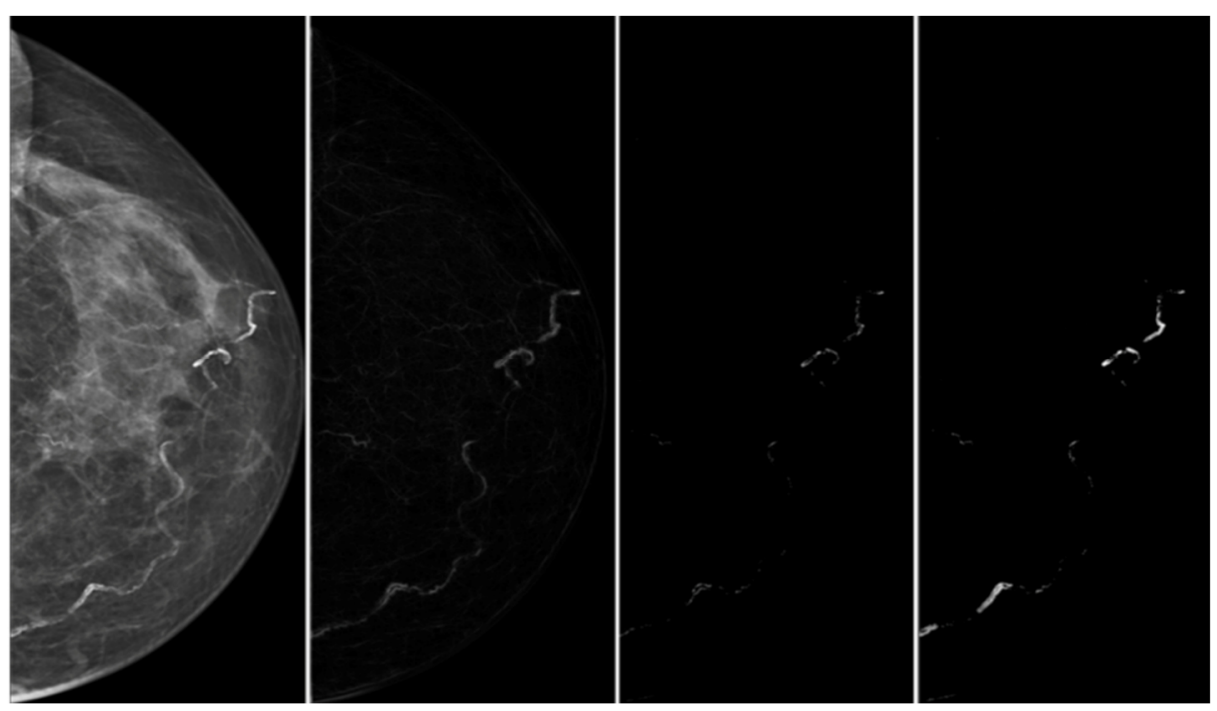

Fig. 3. Full comparison of image through all steps of the proposed method. 
Two types of classification were carried out in the present work; an estimation of the exact grade of calcification and an estimation of the severity of calcification (high or low). For the measure severity, the four BAC grades were divided into two. A grade of 1 or 2 indicates a low severity of calcification whereas a grade of 3 and 4 indicates a high severity. The system could indicate to the radiologist whether the patient has a high severity of BAC or not, and it gives its best guess for the exact grade.

As it can be seen in Figure 4, there is a positive correlation between the grade of calcification and the calculated average white level value after region growing is multiplied by the white intensity of the image after region growing. This shows that for patients with a higher grade of calcification, the calculated value after running all algorithms is also higher. This enables us to identify a pattern between the two elements and be able to classify and quantify the level of BAC in a patient on the base of their calculated white level values.

Figure 5 shows the correlation between the calculated white level after line strength/thresholding and after region growing, and the grade of calcification. Although a positive correlation can still be seen for both graphs, the previous combined region growing, and white intensity graph shows a stronger correlation. Furthermore, multiplying the white level and white intensity numbers together, we are able to obtain more realistic and spaced out values between the four grades of calcification. By calculating this value for each new image, we would be able to compare it with the average values for a specific grade and image number.

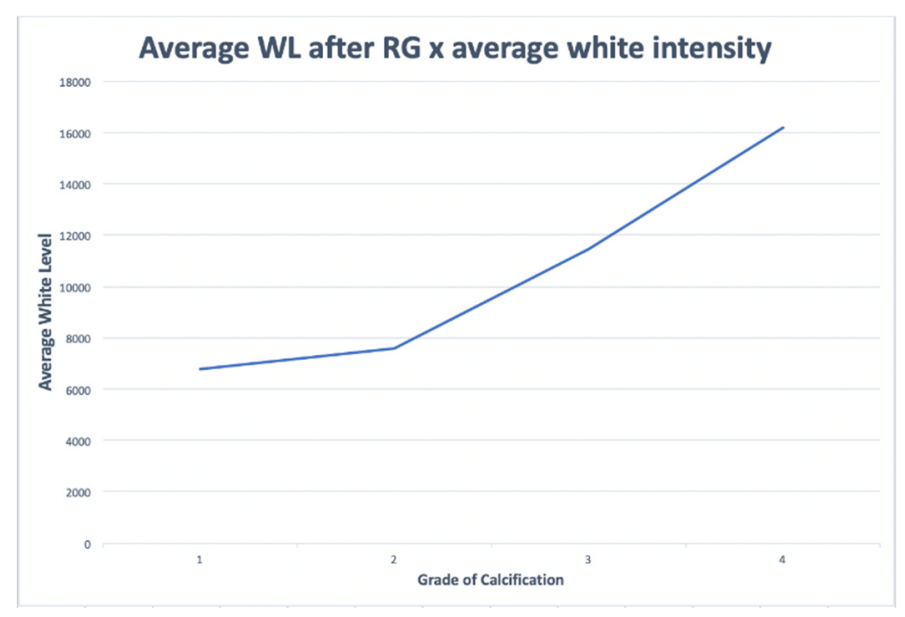

Fig. 4. Graph showing a positive correlation between final white level estimation and the grade of calcification. 


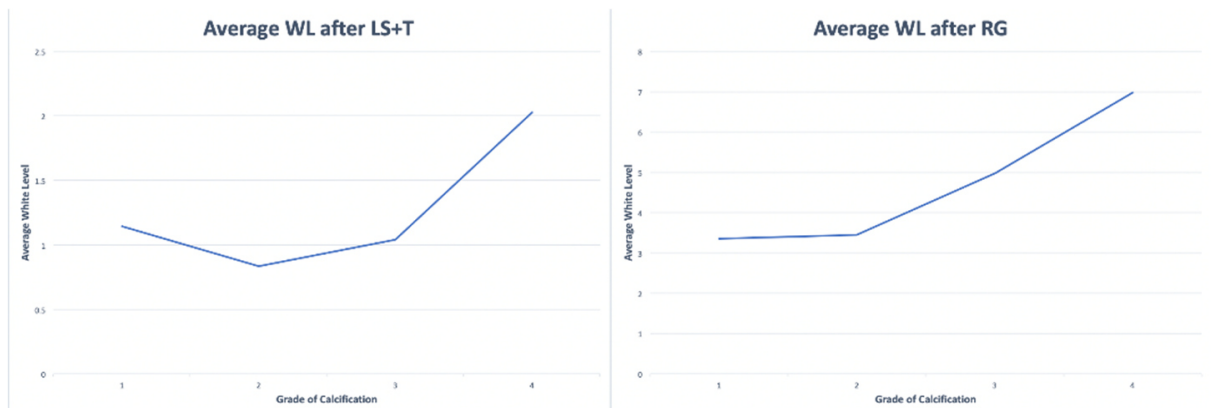

Fig. 5. Left, after Line Strength and thresholding techniques. Right, after region growing algorithm.

The images were reused in order to measure how accurately the system is able to classify BACs, as shown in Figure 6. The highest accuracy of $81.8 \%$ was reached when we wanted to identify whether a patient had a high or low level of calcification by obtaining an average of the four images taken of the patient. By obtaining this average, we are decreasing the chance of having a false positive or false negative result. The second-best accuracy of $68.1 \%$ is reached by obtaining an estimation of the high/low severity of calcification on each individual image. The worst accuracy of $40.9 \%$ was achieved when obtaining an estimate of the exact grade for each image.

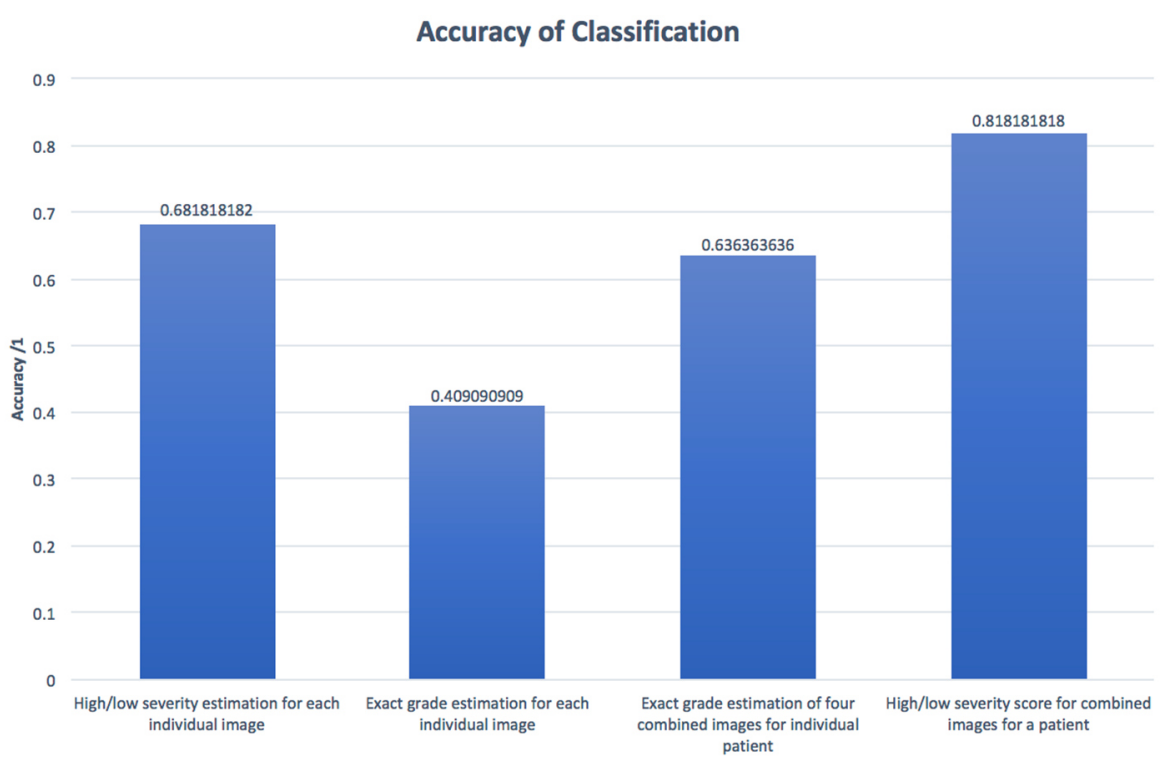

Fig. 6. Classification accuracies achieved. 


\section{Conclusion}

The project explored the possibility to use the breast arterial calcification (BAC) as an indicator of a person being in risk of cardiovascular disease. We presented a system for an automatically quantification and classification of the grade and severity of breast arterial calcifications on mammographic images of patients. The preliminary results made on a consistent dataset provides an adequate degree of accuracy, around $82 \%$, which means that this type of system could be used to assist a radiologist in diagnosing BAC by indicating whether the patient has a high or low severity of calcification. It could be used as an initial automatic screening for all breast cancer patients using their existing mammograms to raise the attention of a doctor if the system estimates a high severity of BAC. This could then be further investigated by a radiologist in order to confirm a result and refer the patient for further treatment. Considering the accuracy reached, the method should be only used be used as an initial aide to a radiologist rather than a standalone diagnosis.

The project lays a solid foundation for further work. There is a substantial opportunity for further work that would have been carried out if not for the constrained project scope. The project set out to research and develop a way to automatically quantity breast arterial calcifications in mammographic images.

\section{Acknowledgements}

This project is made possible by the collaboration with the Derriford Hospital, Imaging Department, and the Peninsula Schools of Medicine and Dentistry in the University of Plymouth, UK, with whom the feasibility of this project has been evaluated.

\section{References}

1. Go AS, Mozaffarian D, Roger VL, Benjamin EJ, Berry JD, Borden WB, et al. Heart disease and stroke statistics. update: a report from the American Heart Association. Circulation.; 127(1): 6-245, (2013).

2. Bairey Merz CN, Shaw LJ, Reis SE, Bittner V, Kelsey SF, Olson M, et al. Insights from the NHLBI-Sponsored Women's Ischemia Syndrome Evaluation (WISE) Study: Part II: gender differences in presentation, diagnosis, and outcome with regard to gender-based pathophysiology of atherosclerosis and macrovascular and microvascular coronary disease. J Am Coll Cardiol; 47(3 suppl): 21-9, (2006).

3. Quyyumi AA. Women and ischemic heart disease: pathophysiologic implications from the Women's Ischemia Syndrome Evaluation (WISE) Study and future research steps. J Am Coll Cardiol.; 47(3 suppl): 66-71, (2006).

4. Leila Mostafavi, Wanda Marfori, Cesar Arellano, Alessia Tognolini, William Speier, Ali Adibi, Stefan G Ruehm Prevalence Of Coronary Artery Disease Evaluated By Coronary Ct Angiography In Women With Mammographically Detected Breast Arterial Calcifications. PLoS One 9;10(4):e0122289, (2015). 
5. Moradi, Maryam, Atoosa Adibi, and Mehdi Abedi. "Relationship between breast arterial calcification on mammography with CT Calcium scoring and coronary CT angiography results." Advanced biomedical research 3 (2014).

6. Nava, Barba, Sendra, Rebollo, Quantification of Vascular Calcifications on Digitized Mammograms, Volume 6136 of the series Lecture Notes in Computer Science pp 183-190, (2010).

7. Cheng, Jie-Zhi, et al. "Detection of arterial calcification in mammograms by random walks." International Conference on Information Processing in Medical Imaging. Springer Berlin Heidelberg, (2009).

8. J. Ge, B. Sahiner, Y. Zhang, et al. Automated detection of breast vascular calcification on full-field digital mammograms. In The International Society for Optical Engineering. 9. C. Iribarren and S. Molloi. Breast arterial calcification: a new marker of cardiovascular risk? Current Cardiovascular Risk Reports, (2013).

10. P. A. H. Van Noord, D. Beijerinck, et al. Mammograms may convey more than breast cancer risk: Breast arterial calcification and arterio-sclerotic related diseases in women of the dom cohort. European Journal of Cancer Prevention, (1997).

11. R. Zwigelaar, S. Astley, et al. Linear structures in mammographic images: Detection and classification. IEEE Transactions on Medical Imaging, (2004).

12. M. Petrou and P. Bosdogianni, Image Processing the Fundamentals, Wiley, UK, 2004. 